the specific impact of the HFVC service during the calendar year 2020 to analyse in particular the value of the service in providing remote specialty care without the need for onward hospital referral.

Method Data including demographics, co-morbidity, medication, frailty, reason for referral, and the impact of the HFVC on what the GP would have done in the absence of this service, were prospectively collected on all cases discussed in HFVC during 2020. Descriptive and frequency analyses were performed using SPSS V.27 [figure 1].

Results The HFVC service discussed 317 cases during 2020. This was a decline from 452 in 2019, likely due to the reduced patient interaction with the GP. Of these cases $(53 \%$ male, 76.8 11.1 yrs.), 205 (65\%) were new case discussions while the remainder represented review or follow-up discussions. Referrals were dominantly from Dublin (35\%), Wicklow (29\%) and Carlow/Kilkenny (27\%). The population in general was vulnerable (Frailty scale $3.8 \pm 1.5$ ), multi-morbid (mean $7 \pm 3.4$ comorbidities) and on polypharmacy (10.4 \pm 4.9 medications). The dominant reason for consultation was assistance with diagnosis (70\%) and the remainder was related to management or investigation interpretation. The greatest impact of the HFVC was a dramatic reduction in the need for onward referral to the hospital outpatient department, either the general cardiology service or the heart failure service. Without the VC service $82 \%$ of the group would have been referred, in contrast to only $11 \%$ requiring hospital outpatient services after the VC consultation, with $72 \%$ now being managed in a shared GP-virtual specialty strategy. The intervention also prevented 3 patients being referred to the ED/AMAU services (figure 2).

Conclusion The HFVC, an already established platform to enable the transfer of aspects of $\mathrm{HF}$ care to the community, was particularly effective during the COVID-19 pandemic in 2020. In providing online, real time specialist advice to the GP, referral to the hospital outpatient service was reduced by 230 referrals, a more than 8 -fold reduction in the year. This was observed in a frail, elderly, multimorbid population who would have been particularly at risk to virus exposure if travel to a hospital setting had been necessary. This experience further underlines the benefits of this platform for care delivery and should be encouraged and developed as a management tool to other cardiovascular problems and indeed other chronic illnesses.

\section{ANALYSIS OF TIMING OF PATIENTS REFERRED TO A NATIONAL TERTIARY ADVANCED HEART FAILURE CLINIC}

N Starr, L Murphy, G Giblin, C Tracey, C Howley, E Kavanagh, J O'Neill, N Mahon, J McGuinness, A Kinsella, E Joyce. Mater Misericordiae University Hospital, Dublin, Ireland

10.1136/heartjnl-2021-ICS.39

Background A hub and spoke healthcare model for national heart failure (HF) management is encouraged internationally. At the heart of this model is the necessity for recognition of patients developing advanced heart failure. Our aim was to understand referral patterns to a National Advanced HF clinic including triggers for referral and burden of disease at review. Methods Patients reviewed at the National Advanced HF clinic between May 2019 and February 2021 were included in an advanced HF registry. Patient outcomes included immediate hospital admission, mechanical support, heart transplant and death. Information such as New York Heart Association (NYHA) class, ESC 2018 'I need help' parameters and 'total HF hospital admissions in the previous year' were documented.

Results There were a total of 56 patients included in the registry of which $28.6 \%(n=16)$ of patients required immediate admission. Of the admitted group, mean NYHA class was $3 \pm 1.1$ compared to NYHA class $2 \pm 0.8$ in those not admitted $(\mathrm{p}<0.001)$. The mean number of 'I need help' markers in the admitted group were significantly higher $(6.2 \pm 2.4 \mathrm{Vs}$ $2.8 \pm 2, \mathrm{p} \leq 0.001)$ and frequency of hospital admissions in the previous year were double that of the not admitted group $(2.3 \pm 1.8$ Vs $1.1 \pm 1.2, p=0.03)$. Of those admitted the 2 main reasons for referral were 1 . intolerance of neurohormonal agents (40\%) and 2.>1 HF hospitalisations (40\%). Ultimately $75 \%$ of those admitted required inotropes and $43.8 \%$ $(n=7)$ went on to have a ventricular assist device (VAD) (3 CentriMag devices and 4 durable LVADs). In total $56.3 \%$ $(n=9)$ were listed for inpatient heart transplant. Of those 3 patients died on the active waiting list (2 CentriMag devices \& 1 on inotropes). A total of $18.9 \%(n=3)$ proceeded to inpatient heart transplant. In the admitted group the mortality rate was $37.5 \%(n=6), 67 \%$ of which occurred during the index admission. This overall death rate was significantly higher than that of the not needing admission group $(37.5 \%$ Vs $5 \%, \mathrm{p}=0.03)$.

Conclusions This study signifies real world data surrounding advanced HF referrals to a national tertiary centre. It highlights the magnitude of patients referred at a significantly advanced stage reflected by almost a third requiring immediate inpatient admission. This translated to a significantly higher mortality rate within this cohort. Importantly, the mean NYHA class was 1 point higher in the admitted group. Although this alone cannot be used to trigger patient referral to an advanced HF clinic, as it is used universally, transition from class 2 to 3 should prompt a review of the patient using a model such as 'I need help' to question the need for referral. Notably of those admitted, $80 \%$ of referrals were triggered by only 2 markers within this model suggesting the usefulness of these 2 parameters for identifying a particularly advanced group of HF patients. Further work is needed however to evaluate which parameters will identify patients at an earlier stage of disease.

\section{IS AN NTPROBNP SALIVA TEST PAVING A NEW AVENUE FOR DIAGNOSIS AND THERAPY MONITORING OF HEART FAILURE PATIENTS - INSIGHTS FROM THE KARDIATOOL STUDY}

${ }^{1} \mathrm{~N}$ Glezeva, ${ }^{1} \mathrm{~J}$ Gallagher, ${ }^{2} \mathrm{M}$ Ledwidge, ${ }^{1} \mathrm{~K}$ McDonald, ${ }^{3} \mathrm{C}$ Watson. ${ }^{1}$ St Vincent's University Hospital and University College Dublin, Ireland; ${ }^{2}$ Heartbeat Trust, Dublin, Ireland; ${ }^{3}$ Queens University Belfast, Belfast, UK

\subsection{6/heartjn|-2021-ICS.40}

Introduction There is ample room for improving the usability of risk prediction tools for the advancement of heart failure (HF) management. New biomarkers and testing technologies, which can diagnose HF, monitor therapy and provide information related to the subsequent risk for adverse events or mortality of the affected patients, may drive this change. Blood NTproBNP is a well-established biomarker for diagnosis and monitoring of HF. Saliva NTproBNP has 